\title{
PHYSICAL ANALYSIS OF THE MECHANISM OF CELL DIVISION IN SEA-URCHIN EGGS
}

\author{
$\operatorname{AUTHOR}(\mathrm{S})$ :
}

Hirota, Takeo

\section{CITATION:}

Hirota, Takeo. PHYSICAL ANALYSIS OF THE MECHANISM OF CELL DIVISION IN SEAURCHIN EGGS. PUBLICATIONS OF THE SETO MARINE BIOLOGICAL LABORATORY 1953, 3(1): 109-122

\section{ISSUE DATE:}

1953-07-30

URL:

http://hdl.handle.net/2433/174457

RIGHT: 


\title{
PHYSICAL ANALYSIS OF THE MECHANISM OF CELT. DIVISION IN SEA-URCHIN EGGS ${ }^{132) 3)}$
}

\author{
TAKEO HIROTA* \\ Zoological Institute, Kyoto University \\ With Plate III and 2 Text-figures
}

\section{Introduction}

The history of studying mechanism of cell division has already passed more than a hundred years, but nothing has been clarified physico-chemically. Numerous observations in various cases have shown us contradictions and confused us by their complications. Up to this day, even the term "pole" in the dividing cell has never been known about its nature. Of course, no successful research has been done to know the cause by which the cell division is induced.

F. Schrader (1) has pointed out that the hypothesis which intends to explain each phase of cell division by force is nothing but the expostre of our ignorance about the physico-chemical nature of the cell, because even one of the reasonings on each phase can hardly be explained without any assumptions.

Indeed, numerous observations will be precious on some points of view, but if one can not understand the essential properties of component substances of the cell, the physico-chemical study of the cells will have no meaning. Therefore, we would not depend upon the view-points derived from the bases of the classical hypothesis. A new scientific method will have to be discovered.

Certainly, microscopical observations can not suggest us directly the forces by which the movement of chromosomes or other cell elements takes place.

But, we cannot atse the microscope because the size of the cells fits their visible ranges, so that the nature of the observing method must be reconsidered.

* Present address--Physiological Laboratory, Faculty of Medicine, Kyoto University.

1) Contributions from the Seto Marine Biological Laboratory, No. 204.

2) Reported at the general Meeting of the Genetic Society of Japan, Oct. 13, 1950, held at Tokyo University.

3) Report of the Nippon Institute for Scientific Research on Pearls, No. 21.

Publ. Seto Mar. Biol. Lab., III (1), 1953. (Article 8) 
In general, the experimental method defines the meanings of data gained, such microscopical data tell us only the difference of the refractive index or wave length of light etc..

Our studies on the mechanism of cell division have to be based on the forces derived from a physico-chemical point of view. Then we must determine what methods are preferable to calculate the displacement by forces using only the microscope.

Fortunately, the author has found that a very primitive way to calculate them indirectly is left in some cases. At first, the standpoint on the analysis of the mechanism of cell division was reconsidered as follows.

1) Since fixed figures as artefacts are able to show us nothing about forces, the cells must be observed in a living state.

2) The phase contrast microscope may be the best one for such restricted observations.

3) The moving state must be photographed in continuous series with some intervals in order to make them put in a still state.

4) Microscopical data related with the force are only the changes of the length, in other words " displacement".

The length $(L)$ relates to the volume $\left(V=L^{3}\right)$,

and by Boyr.e-Charles' law, $\quad P V=n R T$

While the room temperature $(T)$ and the medium pressure $(P)$ are kept in constant in these observations, if the partial volume changes $(d V)$ of the cell are observed, it must be considered that the outward pressure $(\boldsymbol{P})$ of the protoplasm against the medium pressure at such points ought to be changed. Originally, the pressure means the thermal molecular movement.

However, a direct measurement of the change of $P$ in these observations would be impossible.

Only the changes of $(L)$ will correlate with the calculation as a result of $P$ indirectly.

Actual principles of calculation will be shown in the following pages.

It has to be borne in mind that the changes of the visible structures are due to the cause derived from physico-chemical phenomena in colloidal dimensions and not in microscopical ranges. Therefore, it is obvious that this method cannot also give us the chance to intend the causal analysis of the cell division.

If actual data do not match with the result of the calculation, it is also obvious that this method must be abandoned.

Here, we shall have to determine what kinds of analysing method should be 
possible. However, for this purpose, it is necessary to know the behavior of the cell itself in kinetic stages.

\section{Preliminary Observation for the Bases of the Physico-Mathematical Analysis}

Following three facts were observed preliminarily.

1) After the insemination, two main axes of the eggs changed their length gradually from round to ellipsoid until the deepening of the cleavage furrow began.

2) When the changes of both axes are proceeding, the separation of both centrospheres went on in the cell and then the cleavage took place along the short axis.

3) The cleavage surface had never been found as a plane. It was always shown as a bi-sphere just like two balls attached. Then the attached area of both daughter halves increased. While, in this phase, the second separation of the centrosphere had already proceeded, and moreover three axes of daughter spheres had gradually changed, it must be considered as the second ellipsoidal elongation. Then the second cleavage took place's.

This process was confirmed three times by different eggs of sea-urchins such as: Anthocidaris crassispina ('48), Tripneustes gratilla ('49), and Mespilia globulus ('50).

Above observations are able to understand as follows.

1) Some unknown internal changes took place in the egg by the fertilization.

2) The surface tension of the egg by which the shapes are held decreases; i.e. the round surface tends to the ellipsoidal surface with two different curvatures.

3) This surface deformation is naturally expected to give arise the migration of the inner elements. The main intracellular migrating element was the separation of the centrospheres.

4) It is easily supposed that the surface, decreasing its volume around the short axis, have to be unstabilized by its concentrated colloid substances. So, it will be understood that the bi-spherical furrow around the short axis appears. As long as the internal changes continue, the same process will have to be repeated. Accordingly, the second cleavage will take place in the same manner.

If these observations and understandings are correct, we can attain the following physico-chemical presumption.

1) Ref. E. B. HARVeY and G. I. LAvin, Exp. Cell. Res., Vol. 2, No. 3., p. 398, 1951. 
If the volume of the system is kept in constant, the deformation from a sphere to an ellipsoid will increase the surface area.

Thus unit surface free energy must be in constant because the medium pressure remains in constant.

Therefore, the total surface free energy tends to increase.

Would it be possible?

On the contrary, if a decrease in the surface free energy occurs, the increase of the surface area must take place. The ellipsoidal surface deformation must be a spontaneous increase of the surface area to repair the decrease of the total surface free energy.

No doubt the cleavage from a large sphere to two small daughter spheres is the latter case.

\section{Observations and Results}

Microscopical Study.

Eggs used in this study are almost transparent under an ordinary microscope. But under applied phase conditions ${ }^{1)}$, numerous small granules were recognized. In the unfertilized matured eggs, the nucleus was hardly seen in this phase condition due to its diffuse contrast.

Several minttes after the insemination, a dark contrasted region with no structures appeared clearly at the center ${ }^{2)}$. Then this dark contrasted region surrounded by the bright contrasted particles was elongated towards the long axis accompanied with the gradual surface deformation. Probably, this would be an initial spindle formation. As the low power of the microscope was used, the behaviors of other details could not be clearly observed.

This observation is not the morphological one in which the details of structures have to be studied. While the main problem of this study was the displacement, the oil immersion lens was not used for fear that it might not give us the data correctly by its suppression ${ }^{3)}$. But the details do not make any difference to me. The dark constrasted portion was enough to indicate the position of the cell element.

As the elongation of this region was going on, the midregion of it lost its contrast and only the terminal part of it was found to remain clear. Bright contrasted granules around this terminal part seemed to be arranged radially

1) $40 \times 15$ Bright Medium, Green filter Tiyoda Co. Tokyo.

2) This is an interesting phenomenon in colloid system in respect to the views of coacervates by T. HIROTA.

3) It is expectable that the radial arrangements of the cytoplasmic granules by suppression of the cover glass will occur. The astral figure will be intensified. 
somewhat strained. It is supposed that these shapes will be identical to the centrosphere. The cell became the more ellipsoidal, the more the distance between centrospheres was seen to separate. When the axial ratio of the ellipsoidal egg attained about 8:11, the surface around the short axis became unstable and then the cleavage furrow began to deepen.

As was described in the preliminary result 3 ), it was confirmed that the cleaving surface was not a plane but a bi-sphere. Perhaps, this phenomenon would have been observed by many workers repeatedly, but no one suggested its physical meanings.
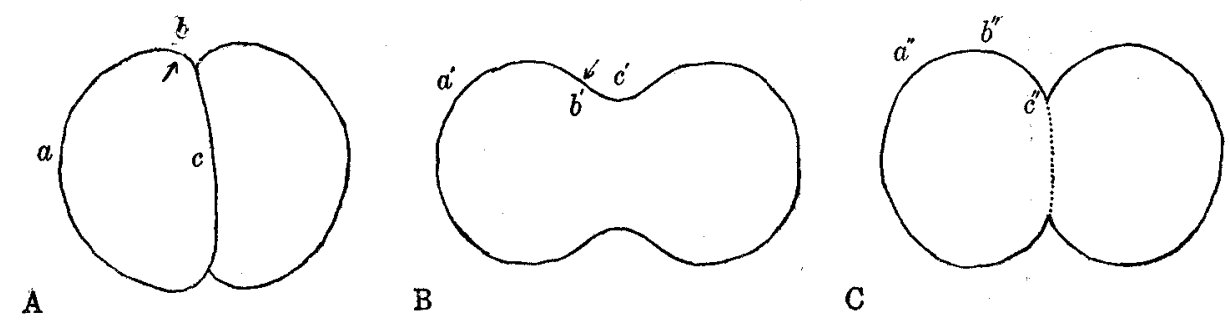

Fig. 1. The three types of the cleavage surface.

A. (Bi-cup type). The curvatures of $a, b$ and $c$ are not equal. It is difficult to occur as far as its surface tension is decreased or suppressed.

B. (Bi-lobic type). The curvature at $a^{\prime}$ is positive and that at $c^{\prime}$ is negative $b^{\prime}$ is the bending point. Their surface tension at these three points must differ each other. This is also difficult to occur.

C. (Bi-spheric type). $a^{\prime \prime}$ and $b^{\prime \prime}$ are almost equal in their curvatures but $c^{\prime \prime}$ is a discontinuous point. Furrowings is fascilitated to occur at such unequilibrium points.

None of the three observations showed such a cut plane or a pulled bi-lobic surface, but always showed a bi-spherical surface (See Fig. 1, A and B). The term "cleavage furrow" does not fit to express this phenomenon exactly. The term "the cleaving spherical surface" and "the cleaved attaching plane" had better be used separatedly (Fig. 1, C). This is an important observation to suggest the physical properties of the cleaving surface.

Reconsideration for Measurements and Theoretical Calculation.

In general, the volume decrease will occur due to the fertilization or other physico-chemical treaties.

Then we would consider the volume contraction on the short axis. If the slight volume contraction around the short axis takes place, the next inner layer must contract in the same way more or less in the higher rate. Concentrically, these contraction must affect to the central portion, and cell elements initially located at the central region have to move towards the long axis. In these 
cases, the mean migrating direction of each particle in the cell must be perpendicular to the moving surface after the sign of $P$ in GrBB's adsorption equation (2) (3). These considerations make the calculation of the displacement of internal elements corresponding to the surface deformation possible.

The displacement ratio is proportional to $r^{\prime-2}(4) ; r^{\prime}$ indicates the distance from the center of the surface curvature to the observing point on the main axis ${ }^{11}$.

This presupposition seems to be very rough, but it must occur decisively, if no disturbances arise in these processes.

For the determination of this view-point, the observing points were taken to answer the next questions.

1) Does the volume decrease really occur in the process of the cell division?

2) Do the changes of the length of both main ellipsoidal axes correlate timely with each other throughout the whole process?

3) Can the displacement of internal structural elements be calculated from the surface deformation?

4) Are the deepenings of the cleavage furrow independent from 2) and 3)?

The calculation was only facilitated with the point on both axes, but on the other portion it is permitted only as an approximation or an inference from the former.

For the above cited measurement, materials and methods were selected as described in the next section.

Materials and Methods.

A) Materials. Sea-urchin eggs (Mespilia globulus).

B) Method and condition. Room temperature $26^{\circ} \mathrm{C}-27^{\circ} \mathrm{C}$.

(1) Observing apparatus. Phase contrast microscope (Tiyoda Co. Tokyo)

ob. 20 B.M. oc. $15 \times$

Light source. $200 \mathrm{~W}, 100 \mathrm{~V}$.

Filter for heat. $10 \mathrm{~cm}$ water filter.

Filter for wave length, green filter (Tiyoda Co.)

Intensity regulator. $10 \mathrm{am}$. slide dug.

Camera. Leica, Fuji F. panchro $35 \mathrm{~mm}$.

(2) Preparation. Eggs and sperm,-Natural breeding.

Insemination. Under the phase contrast microscope by an usual way.

1) $-\partial P / \partial A=\partial \gamma / \partial V \mathrm{dv}=\mathrm{ds} r^{2} / r^{\prime 2}$

The sign of the term $\partial P$ gives the direction of the pressure. ds, surface deformation. $d v$, displacement of the marked elements. $r$, radius of the surface curvature. $r^{\prime}$, distance from the center to the marked elements. 
(3) Continuous photographing. Exposure, 1 sec.

Timing, 30 sec. $\sim 1 \mathrm{~min}$. for the quick phase.

$5 \mathrm{~min} . \sim 3 \mathrm{~min}$. for the slow phase.

The usual observations were made at $30 \mathrm{~V}$. $(60 \mathrm{~W})$. It was photographed with above-cited time intervals from the insemination to the cleavage.

C) Measurement. The long axis $(a)$, short axis $(b)$ of the deforming eggs and the distance between both centrospheres $(c)$ were measured in each photograph. And moreover, the cleaving depth of furrows was measured $(d)$. But the jelly coat layer was omitted in this measurement.

D) Calculation. Radii of the surface curvature at the top of the long axis are given in the next equation 13.

$$
r=b^{2} / a
$$

Calculations for the position of the centrosphere $\left(c^{\prime}\right)$ are given as follows.

$$
c^{\prime}=a e=a-r
$$

Thus $c$ and $c^{\prime}$ were compared.

E) Other experimental condition. The influence of the light was carefully minimized as sometimes the process was reversed by the higher temperature.

The effect of the the phase contrast was not at its best conditions. As the breeding condition was very difficult, it was necessary to keep the temperature of the sea water below $27^{\circ} \mathrm{C}$..

\section{AdDitional Observations.}

If the internal changes which give rise to the decrease of the surface free energy are continued, the bi-spheric daughter halves have to become attached bi-ellipsoids and may be appeared as a bi-cup shape.

Surprisingly, it was found that the second separation of the centrosphere has been proceeded in each attached bi-ellipsoid as seen in the first cleavage. If the third axis perpedicular to the observing plane in the bi-cup stage is able to observe from the pole, we shall find them changing.

It is evident from these observations that the first and the second division may have been proceeded by the same mechanism.

However, these are the reports of the observation which was gained only by eyes. Uncertainties and subjectivities cannot be swept off especially in morphological studies like this. Moreover, photographic evidence will sometimes

1) The radius of the surface curvature is given by the next equation.

$$
\begin{aligned}
& y=\sqrt{b^{2}\left(1-x^{2} / a^{2}\right)} \text { but, } r=\left(1+y^{\prime 2}\right)^{3 / 2} / y^{\prime \prime} \text { so, } r=\left(a^{2}-e^{2} x^{2}\right)^{2 / 2} / a b \\
& \\
& \text { but, } e=1-b^{2} / a^{2}, \quad \therefore r=b^{2} / a, \text { at the top of the long axis, } \\
& c^{\prime} \text { is given by } c^{\prime}=a e=a-r
\end{aligned}
$$


be equal to the picture card which was brought back from an excursion trip.

Then, we must offer the result of the physical analysis from the foregoing principle $\mathrm{D}$ ). Of course, this physical treatment must demonstrate directly the facts which were observed and understood in the preceding manner.

\section{Physical Measurements and Calculations.}

Main difficulties occurred from the differences of the egg maturity and of the durating time of dividing processes and moreover from the inconvenient, random occurrences of the cleavage plane. Each of them disturbed the determination of the starting point, timing and physical measurements, respectively.

The maturity was then assessed by fully rounded shape and the diffuse contrast of the nuclear region.

Since the time factor was eliminated from the calculating equation, the time intervals did not possess the absolute physical meanings. This is the most important fact in this analysis.

Gradual changes of dividing shapes were photographed by the adaptable timing to the proceeding velocities of each phase. Mainly, it was considered to

Table 1. Changes of the long and short axis, measured and calculated distances of the centrosphere and the depth of the cleavage furrow.

\begin{tabular}{|c|c|c|c|c|c|}
\hline $\begin{array}{c}\text { Photo } \\
\text { No. }\end{array}$ & $\begin{array}{l}\text { Long } \\
\text { axis } \\
\text { (a) }\end{array}$ & $\begin{array}{l}\text { Short } \\
\text { axis } \\
\text { (b) }\end{array}$ & $\begin{array}{l}\text { Distances of } \\
\text { both } \\
\text { centrospheres } \\
\text { (c) }\end{array}$ & $\begin{array}{c}\text { Calculated } \\
\text { distance of } \\
\text { centrospheres } \\
\left(c^{\prime}=a \times e\right)\end{array}$ & $\begin{array}{l}\text { Depth of } \\
\text { cleavage } \\
\text { furrow } \\
\text { (d) }\end{array}$ \\
\hline 1 & 19.5 & 19.5 & 0.0 & 0.0 & 一 \\
\hline 2 & 20.2 & 18.7 & 1.60 & 1.82 & - \\
\hline 3 & 20.6 & 17.8 & 4.60 & 5.28 & - \\
\hline 4 & 21.1 & 17.2 & 6.70 & 7.06 & 一 \\
\hline 5 & 21.6 & 16.6 & 8.50 & 8.89 & 16.4 \\
\hline 6 & 22.0 & 16.3 & 9.60 & 9.94 & 16.0 \\
\hline 7 & 22.4 & 16.3 & 10.00 & 10.53 & 15.0 \\
\hline 8 & 23.1 & 16.0 & 11.70 & 12.08 & 14.0 \\
\hline 9 & 23.6 & 15.6 & 13.20 & 13.70 & 13.5 \\
\hline 10 & 24.0 & 15.7 & 13.70 & 13.82 & 11.9 \\
\hline 11 & 24.5 & 15.8 & 13.70 & 14.32 & 11.3 \\
\hline 12 & 24.6 & 15.8 & 13.80 & 14.44 & 10.0 \\
\hline 13 & 24.6 & 16.0 & 14,20 & 14.45 & 9.4 \\
\hline 14 & 24.5 & 16.0 & 13.70 & 14.45 & 8.4 \\
\hline 15 & 24.4 & 16.4 & & & 11.0 \\
\hline 16 & 23.7 & 16.5 & & & 13.4 \\
\hline 17 & 23.2 & 16.7 & & & 14.2 \\
\hline 18 & 23.0 & 17.7 & & & 14.8 \\
\hline 19 & 22.5 & 16.5 & & & 15.6 \\
\hline 20 & 22.3 & 16.5 & & & 15.6 \\
\hline 21 & 22.5 & 16.3 & & & 13.8 \\
\hline
\end{tabular}


catch the observing points continually.

Thus, the moving series of the dividing shapes were photographed.

Measurements were made from each photograph enlarged 2.5 times. The data meastured and calculated are shown in Table 1. In this Table, a), b), c), $c^{\prime}$ ) and d) are the values discussed in the previous section C) and D).

The whole process finished in 30 45 minutes. No. $1 \sim 7$ are the ellipsoidal surface deformation. No. 8 14 are the cleaving phase, the calculations in this phase are not accurate. No. $15 \sim 21$ are a process of the second cleavage.

All the values are relative ones but not of actual length.

The interrelations of these values cited in the Table 1 are shown in Fig. 2.

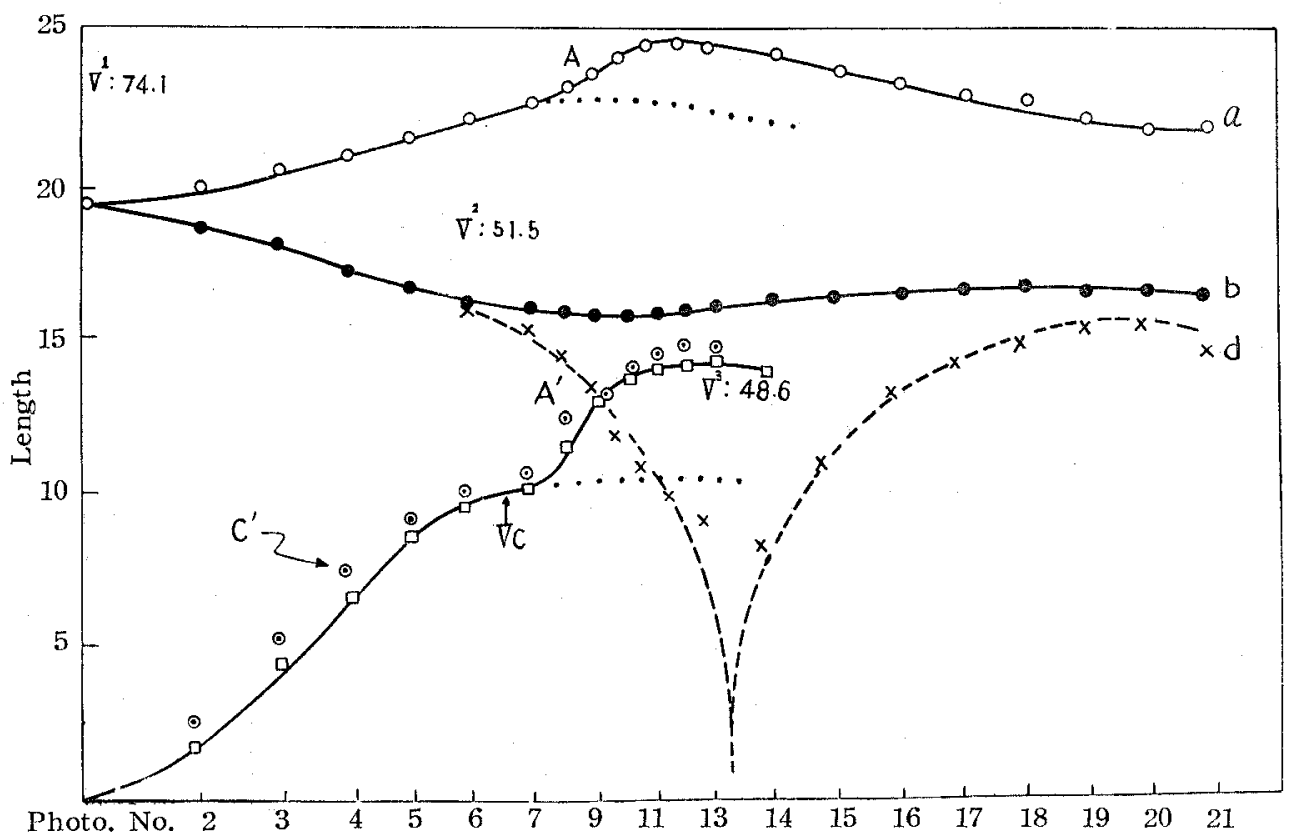

Fig. 2. Interrelation curves of various length shown in Table 1.

(a) Long axis, (b) Short axis, (c) Centrosphere observed, ( $c^{\prime}$ ) Centrosphere calculated, (d) Attached area in cleaving surface, (V) The volume calculated from (a) and $(b) . A$ and $A^{\prime}$ show the irregular elevation of the curve.

If the pushing effect by bi-spheres does not appear, the dotted lines will be the normal curve expected.

Total volume of the egg in the cleavage process was calculated when it had the most regular shape. The values of the initial egg, of the ellipsoidal shape elongated maximally and of the bi-spheric shape, were given as $74.1,57.6$ and 48.6 respectively.

Obviously the values have decreased. The volume calculation was made by 
the formula $4 / 3 \pi a b^{2}$.

Changes of the long axis $(a)$ and short axis $(b)$ showed a good correlation with each other timely. But the jelly coat layer was excepted from this measurements, because it was suggested that they may be an excreted layer (6).

We were particularly interested in the relation between the changes of both axes and the movement of the centrosphere. The displacement of the centrosphere was calculated through the next principle.

In the imaginal corn formed by the volume increasing surface and its center, the product of the surface displacement $(d s)$ and its surface area is equal to that of the expected migration $(d v)$ of the marked element and its localized surface area in the corn. So we will gain the next equation.

$$
\begin{array}{ll}
\theta \pi r^{2} d s=\theta \pi r^{\prime} d v & \theta ; \text { solid angle of the corn. } \\
\therefore d v=d s r^{2} / r^{\prime 2} & r ; \text { radius of the surface curvature. } \\
& r^{\prime} ; \text { distance from the center to the marked element. }
\end{array}
$$

But we found in this case, $r^{\prime}=c-(a-r) \div 0 \quad \therefore c^{\prime} \div(a-r)=a e$

So $c^{\prime}$ is equal to the focus of the ellipsoid. Therefore, we are able to calculate the approximate position of the centrosphere shown in Table 1.

This is an important and surprising phenomenon in which the mathematical treaties are held strictly about the pole. It has been also found that such treaties are applicable in some other cases more complicated (4).

The irregular elevation $A$ and $A^{\prime}$ in Fig. 2 make a good correspondence timely. The deepenings of the cleavage furrow $(d)$ correspond to these elevation too.

The $A^{\prime}$ in the separation curve of the centrosphere is identical to the "pause" termed by H. RIs (5) in his anaphase study. The stage to the pause is the so-called anaphase and another stage after the pause must be included in the so-called telophase.

About the deepening of the cleavage furrow $(d)$, it will be analysed in another paper. No. $7 \sim 13$ show the depth of the bi-spherical cleavage furrow and No. 13 20 show the increase of the attached area of both datighter halves. Both ellipsoidal axes after No. 12 ( $\mathrm{a}$ and $\mathrm{b}$ ) have shown the reversal change. Of course, the third axis has been shortened and the second separation of the daughter centrosphere has also took place.

\section{Conclusion}

1) In general, we can not suggest the cause of cell divisions from these microscopical studies because the forces can not be recognized directly. 
2) The about $30 \%$ volume decrease was observed in the process of the cell division.

3) The ellipsoidal deformation of the cell was ascribed to the decrease of the surface free energy.

4) The migrated distance of the cell element from the initially located position was calculated approximately from the changes of both axes of the ellipsoid and surface deformations. The results of calculations and measurements showed a good coincidence. In this case, the centrospheres were marked as the displacement elements in the cell.

5) The centrospheres appeared closely at both foci of the ellipsoidal egg. The pole of the dividing cell is not a mysterious center of the imaginal force but a physical center of the migrating cell elements at least.

6) It must be remarked that the cleaving plane showed always an attached bi-spheric type and never cleaved with a bi-cup shape. The latter is the following phase after the former.

7) The pause was observed in the migration of the centrosphere but it is able to be ascribed to the bi-spheric pushing effect.

\section{Discussion}

No more discussion about each part will be necessary, because each part has been explained with no assumptions. If any presumptions were introduced, the works should be done to demonstrate the assumption itself.

Although the late numerous observations are very valuable, the various explanations about the cell division derived from the imaginal force which was impressed from the morphological changes can not but be hypothetical.

Such hypothesis had never been proved their reliability. It may be inevitable, because the physico-chemical constructions of the cell were not understood enough.

The author was very interested in the anaphase study by H. Ris (5), since some conclusions were made in the same way (3) (4).

Any other data except displacement are not responsible as long as we are dealing with the force with the microscope in the living cell.

The migration force of chromosomes has been attributed to the imaginal activity of some structures in the passive state in the classical hypotheses.

Recently, an autonomous migration of the chromosomes has been suggested by some authors (1). The late hypotheses seem to have been asked the reconsideration by these observations. However, what method will be available 
to detect whether the migration must be passive or autonomous?

Since the author had experienced that each case showed the gradual surface deformation, it had been studied that a reasonable spontaneous displacement of cell elements is accompanied with this deformation.

The cell membrane must be primarily treated as a physical boundary surface as far as the surface tension is effective to hold its shape (7), although a precipitation membrane or a denatured membrane will occur by its changed conditions.

As was described above, it is obvious that the bi-spherical cleavage surface may be a good evidence for the surface tension. It has been. shown that the gradual surface deformation and the bi-spheric cleavage also take place in other cases (8).

Surface deformations suggest us the decrease of surface free energy.

But our microscopical studies are limited in respect to the changes of internal colloidal state by which the decrease of surface free energy occurred.

Then, if one portion of the cell surface which is composed of numerous colloid particles displaces in a slight distance perpendicular to the surface, the adjacent sublayer of this portion must be displaced with it in order.

If this expectation has been realized in the cell, the calculation which was described in the preceding section must be established.

As well as in this study, in the spermatogenesis of grass-hopper-the spindle elongation, the chromosome arrangement in metaphase, the chromosome migration in the anaphase, the behaviors of the mitochondria - there is found the fact that the same calculation is closely established (4) (9).

But the appearance of the chromosomes in the prophase, the disappearance of them after telophase and the formations of aster or fertilization membrane are the problems of the colloid physical changes $(10)\left(10^{\prime}\right)$.

Therefore, the physical analysis of the coacervation will be able to answer for these problems (10).

Some interesting observations related to these problems were reported by several authors, i.e. surface granules and the fertilization membrane (11), the grantiar strand and astral formation (12), migration of basophilic granules (14) and mitochondrial behaviors (24) morphologically; other observations were made physically, i.e. the astral ray as a stream line (15) and as a streaming or a structual birefringence (13) (16).

On the other hand, the chemical analyses were made: Changes of the DNA contents after the fertilization (17), of amino acid composition of total proteins (18) (23), of free amino acid (19), jelly coat substances (20) and the electro- 
constriction of amino acid by dissociation (21) (22).

These studies may be responsible for studying the cause of cell divisions, if they were confirmed systematically in the physical point of view.

The surface deformation had been pointed out several times, but no one calculated the migration of the cell elements from it.

In this paper, some natures of the dividing pole were characterized from this calculation. Although further investigations should be necessary, it is sure it may be a physical center of the protoplasmic streaming at least.

In addition to this, the reasons of the pause of the centrosphere were suggested from the migration curve of Fig. 2.

If only one reality in the complex natural scientific phenomena was truly analysed, other numerous phenomena will be solved from its same basement.

In conclusion I wish to express my deepest gratitude to the members of the Seto Marine Biological Laboratory who had conferred me suggestions and conveniences in carrying out these experiments and to Professor K. Nakamura who had encouraged on this study.

\section{Summary}

1) The physical analysis of the cell division by some sea-urchin eggs was attempted with the phase contrast microscope and the continuous photographing method.

2) The decrease of the total volume was showed evidently by calculations.

3) Changes of both axes and displacements of the centrosphere were measured when the ellipsoidal surface deformation was proceeding.

4) The results of the calculation about the position of the centrosphere showed a good coincidence with the data measured.

Therefore it was suggested that the cell elements may be displaced physically and some physical characters about the pole and the pause are clarified.

5) The surface of the cleavage furrow showed a bi-spheric shape and the bi-cup shape appeared in the next phase of it.

It was suggested that they may be resulted from a decrease in the surface free energy.

As the basal view-points, it has been held firmly throughout the whole work that the length is the only data gained physically. 


\section{REFERENCES}

1) F. Schrader, Mitosis, Columbia University Press, New York, 1947. Chap. 2, sect. 3, B, c.

2) W. Grbbs, Collected papers, London, 1906. cited by S. SAMEjIMA, Colloid, p. 57, Shôkabô, Tokyo, 1940.

2') Physical chemistry, edited by S. Mizushima Vol. 4, p. 1051, Kyôritsusha, Tokyo, 1949.

3) T. Hirota, reported at Gen. Meet. Jap. Zool. Soc., Oct. 10, 1949.

4) T. Hirota, reported at Gen. Meet. Jap. Gen. Soc., Oct. 13, 1950.

5) Hans RIS, Biol. Bull., 96, pp. 90-106, 1949.

6) E. E JUST, The physiology of cell surface, p. 147, Blakiston, Philadelphia, 1939.

7) H. G. BUnGenberg DE Jong, Protoplasma, 15, p. 110, 1932.

8) K. MJCHEL (The mechanism of cell division) 1941. Imported and introduced by K. DAN, 1950.

9) T. Hirota, reported at Kinki Br. Meet. Jap. Zool. Soc., Nov. 20, 1950. reported at Kinki Br. Meet. Jap. Physiol. Soc., April 23, 1952.

10) T. Hirota, reported at Gen. Meet. Jap. Gen. Soc., Oct. 13, 1951.

$10^{\prime}$ ) T. HrRoTA, Unpublished.

11) Y. ENDo, reported at Symp. Cyto-Chem, (Embryology) held at Kyoto Univ. Oct. 22, 1951.

12) Y. TAdano, Sci. Rep. Tohoku Univ. (Biol.), 16, p. 100, 1951.

13) S. INOUE and K. DAN, Kagaku, 19, p. 111, 1949.

14) I. MotomUra, Sci. Rep. Tohoku Univ. (Biol.), 18, p. 266, 1950.

15) F. Schrader, Mitosis, Chap. 2 sect. 4, C, Aster, 1947. ref. (1)

16) W. T. SchmiDT, Die Doppelbrechnung von Karyoplasma und Metaplasma, Bornträger, Berlin, 1937.

17) G. Schmidt, L. Hecht and S. ThanHauser, J. Gen. Physiol., 31, p. 203, 1948.

18) M. Takanami and Y. Saito, reported at Gen. Meet. Jap. Zool. Soc., Oct. 13, 1951.

19) T. Gustafson, and Maj-Britt HJelte, Exp. Cell Res., 2, p. 474, 1951.

20) L. Monne and S. B. David, J. Exp. Cell Res, 1, p. 477, 1950.

21) F. T. Gucker, W. L. Ford and C. E. Moser, J. Phys. Chem., 43, p. 153, 1939.

22) —_, _ - Ibid., 45, p. 309, 1941.

23) M. TAKANAmi and T. Hirota, In press.

24) T. Hirota, Unpublished.

R. Shimakura, reported at Gen. Meet. Jap. Zool. Soc., held at Tohoku Univ., Oct. $12,1952$.

\section{EXPLANANTION OF PLATE III}

1) The dark contrasted nuclear region appeared soon after the fertilization. 2) An initial phase. 3) Elongated phase of the dark contrasted nuclear region. 4) Initial phase of the bi-spheric cleavage. 5) 6) the completion of the bi-spheric cleavage. 7) Bi-cup phase. Both halves attached gradually after the first cleavage. It will be seen that the second centrospheres have been separated. 
Publ. Seto Mar. Biol. Lab., III, 1 (1953) PLATE III

1

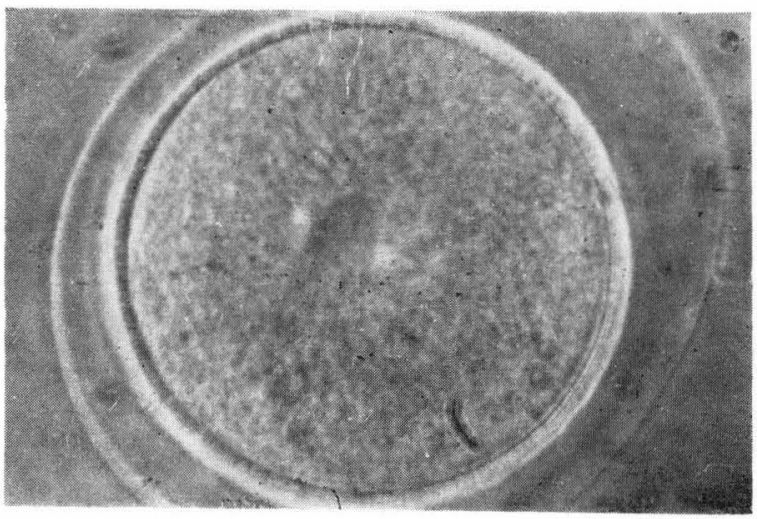

2

5
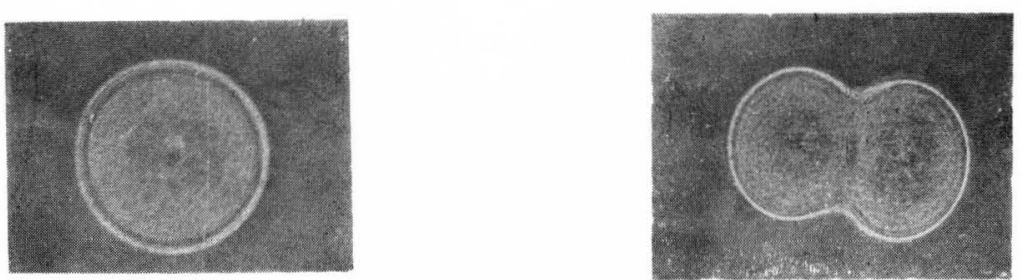

3

6
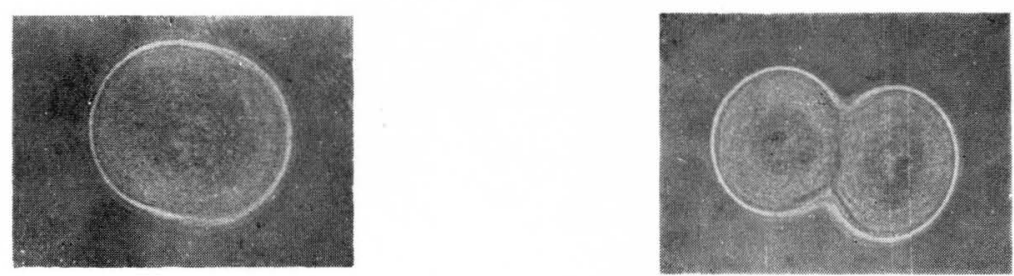

4

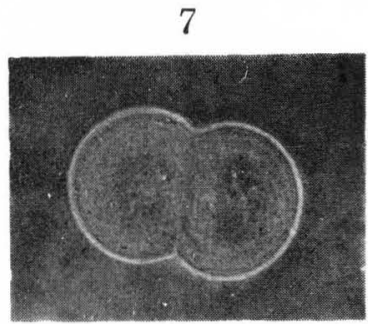

T. Hirota: Physical analysis of Mechanism of Cell Division. 